\title{
PREVALENCE AND CLINICAL SIGNIFICANCE OF CARDIAC MURMURS IN NEONATES
}

\author{
Kumbhojkar A. P1
}

${ }^{1}$ Assistant Professor, Department of Paediatrics, Bharati Vidyapeeth Deemed University Medical College and Hospital, Sangli.

\begin{tabular}{l} 
ABSTRACT \\
\hline BACKGROUND \\
5 to 8 in every 1000 live born babies have a cardiovascular malformation which presents in infancy, but most are asymptomatic at \\
birth. The diagnosis is established only in $50 \%$ of cases by 1 month of age. The difficulties in detecting heart disease at neonatal \\
examination are well known. There are very few studies done so far in developing countries like India in this field. Until preventive \\
measures become available, reduction of infant mortality due to congenital cardiovascular malformations will continue to depend \\
on early recognition of symptoms and signs of serious heart disease in infants and on effective community-wide use of specialised \\
cardiac services.
\end{tabular}

\section{MATERIALS AND METHODS}

Approximately, 1000 infants up to 6 weeks of age were enrolled in study after consent. After echocardiography evaluation subjects with murmur are classified into structurally significant heart lesions, structurally insignificant heart lesions and normal echocardiogram findings. Patent foramen ovale $(<3 \mathrm{~mm})$, peripheral pulmonary artery stenosis, small patent ductus arteriosus $(<2$ $\mathrm{mm}$ ) are classified as structurally insignificant heart lesions. Structurally significant heart lesions are further classified into different congenital heart diseases to establish clinical significance of murmurs.

\section{RESULTS}

Prevalence of cardiac murmurs during routine neonatal clinical examination was 3.9\%. Prevalence of structurally significant heart disease among cases of murmur was $66 \%$. Out of remaining $34 \%$ cases, $21 \%$ had structurally insignificant heart disease and $13 \%$ had normal heart. Family history, significant maternal history, consanguinity and dysmorphic features were statistically significant predictors of underlying structurally significant heart lesions. Early cardiac intervention was required for $16 \%$ cases of structurally significant heart disease.

\section{CONCLUSION}

Unrecognised neonatal heart disease carries a serious risk of avoidable mortality, morbidity and handicap. Early detection of murmur and intervention is important to prevent worsening and complications in cases of congenital heart disease.

\section{KEYWORDS}

Murmur, Neonates, Echocardiography.

HOW TO CITE THIS ARTICLE: Kumbhojkar A. P. Prevalence and clinical significance of cardiac murmurs in neonates. J. Evolution Med. Dent. Sci. 2017;6(89):6227-6232, DOI: 10.14260/jemds/2017/1354

\begin{abstract}
BACKGROUND
Heart is the first organ to form in vertebrate embryos. It has been characterised as the "youngest, most diverse, most fluid, most changeable and the most versatile part of creation." Congenital heart diseases are not fixed anatomic defects that appear at birth but instead are dynamic anomalies that originate in the early embryo, evolve during gestation and change considerably in extrauterine life. Clinical diagnosis of congenital malformation of the heart and circulation represent epitome of applied logic. When correct inferences are drawn from accurate observation, diagnosis emerges with gratifying frequency. One of the most wonderful features of animate nature must surely be a perfect harmony existing between structure and function. No mammalian organ system better exemplifies this principle than the heart. Stethoscope is the oldest cardiovascular diagnostic instrument in clinical use.(1)
\end{abstract}

'Financial or Other Competing Interest': None.

Submission 29-09-2017, Peer Review 28-10-2017,

Acceptance 03-11-2017, Published 13-11-2017.

Corresponding Author:

Dr. Kumbhojkar A. P.,

Department of Paediatrics,

Bharati Hospital, Sangli Miraj Road, Sangli.

E-mail: aniket_kumbhojkar2002@yahoo.com

DOI: $10.14260 /$ jemds $/ 2017 / 1354$
5 to 8 in every 1000 live born babies have a cardiovascular malformation which presents in infancy, but most are asymptomatic at birth. The diagnosis is established only in $50 \%$ of cases by 1 month of age.(2) The auscultation of the heart during routine examination before discharge from the hospital provides an opportunity for early diagnosis of congenital heart disease. Despite this recommendation, routine auscultation has not been subjected to prospective evaluation. The difficulties in detecting heart disease at neonatal examination are well known.(3) The neonatal examination takes place at a time of rapid change within the cardiovascular system as part of adaptation to extrauterine life.(4) These changes may produce murmurs, which can be mistaken for heart disease.(5) Similarly, if transitional changes are slow to occur presentation of congenital heart disease may be delayed, e.g. in case of ventricular septal defect which generally presents at the age of 4 to 6 weeks, presentation will be delayed if there is delay in fall of pulmonary pressure. Detection of a murmur depends on the examiner's skill and experience, the timing and frequency of examination and the conditions under which examination takes place.(6)

Most reports of the prevalence of neonatal murmurs come from early studies,(7) predating echocardiography which has improved the accuracy of diagnosis of congenital heart disease. There is little in published findings that correlates murmurs during the newborn period with 
confirmed anatomical diagnosis. There are very few studies done so far in developing countries like India in this field.(8) Until preventive measures become available, reduction of infant mortality due to congenital cardiovascular malformations will continue to depend on early recognition of symptoms and signs of serious heart disease in infants and on effective community-wide use of specialised cardiac services.(9) This prospective study is designed to determine the prevalence and clinical significance of murmurs heard during routine examination of neonates and the contribution of the neonatal examination to detection of congenital heart disease in neonates.

\section{Aims and Objective}

1. To determine prevalence of cardiac murmurs during routine neonatal clinical examination.

2. To determine the prevalence of structurally significant heart diseases among all murmurs and classify these diseases.

\section{MATERIALS AND METHODS Study Design}

Cross-sectional study in a tertiary care hospital in metropolitan city.

\section{Subject Selection}

Total 977 healthy term newborns were delivered in a tertiary care hospital and those up to 6 weeks of age who come for regular immunisation were enrolled from 01.05.2010 to 01.05.2011 in study after taking informed consent from the parents.

Murmurs are caused by turbulent flow within the heart and great vessels. Murmurs not associated with significant haemodynamic abnormality are referred as innocent murmurs. Heart murmurs are defined by six characteristics: Intensity, quality, location, timing, radiation and conduction. The loudness of a murmur reflects the degree of turbulence. This relates to volume and velocity of flow and not the severity of lesion. Loudness is graded as scale of 1 (barely audible with stethoscope) to 6 (audible without application of stethoscope).(10)

The location of murmur is described as per areas- Upper left sternal border (ULS), Lower left sternal border (LLS), Mitral area (MA), Pulmonary area (PA) and Aortic area (AA). Murmurs are timed according to the phase of systole or diastole. Systolic murmurs are either ejection systolic, pansystolic or late systolic. Diastolic murmurs are either early diastolic, mid diastolic or presystolic in timing. Continuous murmurs were heard during systole and diastole, uninterrupted by a valve closure.

Subjects in the study were screened for presence of murmur with the use of diaphragm and bell of a paediatric stethoscope. All newborns delivered underwent auscultation twice daily during postnatal ward rounds as a routine examination till the time of discharge, which was 3 days in case of normal delivery and 7 days in case of caesarean section delivery.

Details were recorded in a pre-decided proforma, which included history and examination with investigations in the form of chest x-ray, electrocardiography and echocardiography.
Name, age of baby, gestational age at the time of delivery that is either full term or preterm decided by new Ballard score, sex, birth weight measured on electronic weighing scale, order of birth, maternal age, blood group of mother, religion, consanguinity, significant maternal medical and drug history and family history were recorded.

General examination of subjects included heart rate, respiratory rate, peripheral pulses with any discordance between peripheral pulses, NIBP, cyanosis, four limb saturation on and off oxygen using pulse oximeter, signs of congestive cardiac failure, head circumference, dysmorphic features and other congenital anomalies noted.

Cardiovascular system examination is done in detail with reference to heart sounds and nature of second heart sound. Details of murmur type, grade, best heard area, radiation, associated with clicks are recorded along with auscultation of back.

Other systemic examination included respiratory system for breath sounds and adventitious sounds, abdominal examination for liver span and ascites, CNS examination. All the subjects with murmur are subjected to chest x-ray postero-anterior view and standard 12-lead electrocardiographic examination on the same day of detection of murmur before going for echocardiography examination. Electrocardiography analysis is done by senior cardiologist and x-ray reporting is done by senior radiologist. Echocardiography evaluation is done by senior cardiologist in cardiology department using M-mode, colour Doppler, two dimensional using special probe in all those having murmurs in detail using Siemens Vivid 7 echo machine. Various views like subcostal four chamber, subcostal short axis, suprasternal long axis, suprasternal short axis, apical four chamber view, parasternal long axis and parasternal short axis view are used during examination. Structurally significant heart disease is defined as "a gross structural abnormality of the heart or intrathoracic great vessels that is actually or potentially of functional importance."(11)

After echocardiography evaluation subjects with murmur are classified into structurally significant heart lesions, structurally insignificant heart lesions and normal echocardiogram findings. Patent foramen ovale $(<3 \mathrm{~mm}),(12)$ peripheral pulmonary artery stenosis,(13) small patent ductus arteriosus $(<2 \mathrm{~mm})^{(14)}$ are classified as structurally insignificant heart lesions. Structurally significant heart lesions are further classified into different congenital heart diseases to establish clinical significance of murmurs.

\section{Inclusion Criteria}

1. All healthy full term newborns delivered in tertiary care hospital.

2. All babies up to 6 weeks of age who come for immunisation followup.

\section{Exclusion Criteria}

1. Critically ill babies in Neonatal intensive care unit.

2. Preterm neonates and neonates with risk factors for congenital heart disease.

3. Neonates referred from outside and home delivered babies. 


\section{RESULTS}

977 full term neonates were enrolled in this study who underwent routine clinical examination during the study period. Out of these, 427 (43.7\%) were female and 550 (56.3\%) were male babies. Out of 977,38 (3.9\%) had significant audible murmur.

In this study, 38 (3.9\%) newborns out of 977 neonates who underwent clinical examination were found to have murmur. The prevalence of murmurs in routine neonatal clinical examination was found to be $3.9 \%$.

All the 38 cases of murmur were subjected to twodimensional echocardiography. Based on echocardiography diagnosis, cases were divided into Structurally Significant Heart Lesions (SSHL), Structurally Insignificant Heart Lesion (SIHL) and normal; 5 (13\%) cases of murmur had normal echocardiography report, 8 (21\%) patients had structurally insignificant heart lesion and $25(66 \%)$ cases had structurally significant heart lesion. Prevalence of structurally significant heart lesion was $66 \%$.

Out 38 cases with murmur, 18 (48\%) had pansystolic murmur, 17 (44\%) had ejection systolic murmur and $3(8 \%)$ had continuous murmur.

Murmur was best heard at pulmonary area and upper left sternal border in 12 cases each, in 10 (26\%) cases murmur was best heard at left lower sternal border and in only 4 (10\%) cases murmur was best heard at mitral area.

Grade 3 murmur was the most commonly heard murmur during routine neonatal clinical examination. Out of 21 (55\%) babies with grade 3 murmur, 14 (67\%) had structurally significant heart lesion and 7 (33\%) had structurally insignificant heart lesion. Out of $9(24 \%)$ babies with grade 4 murmur, 5 (55\%) had structurally significant heart lesion and $4(45 \%)$ had structurally insignificant heart lesion. Out of $5(13 \%)$ babies with grade 2 murmur, $3(60 \%)$ had structurally significant heart lesion and 2 (40\%) had structurally insignificant heart lesion. Grade 5 murmur was heard in only $3(8 \%)$ babies, all of which were significant. Congenital heart diseases with grade 5 murmur were- 1) Two OS ASD $4.5 \mathrm{~mm}$ and $3 \mathrm{~mm}$, two muscular VSD $2 \mathrm{~mm}$ and 1.2 $\mathrm{mm}$, 2) PDA $4 \mathrm{~mm}$, PFO $2 \mathrm{~mm}$ with pulmonary hypertension and 3) OS ASD $7.2 \mathrm{~mm}$ with PDA of $3.5 \mathrm{~mm}$.

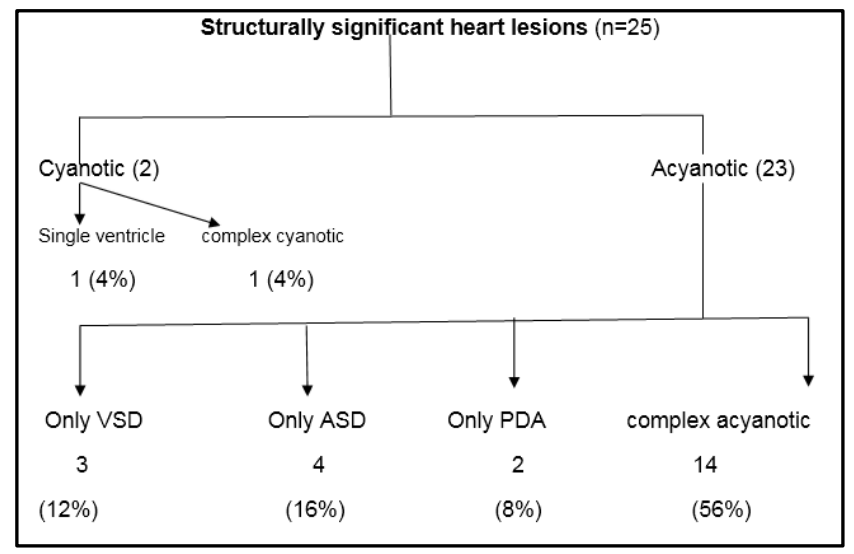

Classification of structurally significant heart lesions $(n=25)$ was done as follows: - $2(8 \%)$ patients had cyanotic type of congenital heart disease- single ventricle $1(4 \%)$ and tricuspid stenosis 1 (4\%). Out of $23(92 \%)$ acyanotic group 3 $(12 \%)$ cases had VSD, $4(16 \%)$ cases had ASD, 2 (8\%) cases had PDA and 14 (56\%) patients had complex form of congenital heart disease. Out of 14 complex acyanotic group 6 (24\%) cases had ASD and PDA as combined lesion, 2 (8\%) cases had VSD and ASD, 2 (8\%) cases had VSD and PDA, 2 (8\%) cases had endocardial cushion defect, 1 (4\%) case had PDA, ASD and VSD, 1 (4\%) case had VSD and pulmonary stenosis.

VSD as an isolated lesion was present in only $3(12 \%)$ cases, but as a part of combined lesions with haemodynamic significance was present in $8(32 \%)$ cases. VSD was contributing to $44 \%$ of significant lesions. Distribution of ASD was $4(16 \%)$ isolated cases and $5(20 \%)$ as a part of combined lesion. Distribution of PDA was $2(8 \%)$ isolated cases and $5(20 \%)$ as a part of combined lesion. Structurally significant heart lesions are independent of location of murmur. Structurally significant heart lesions are independent of grade of murmur. X-ray abnormality is not associated with structurally significant heart lesions. Abnormal ECG is not associated with structurally significant heart lesions. Family history of congenital heart disease was present in $2(5 \%)$ cases among murmur cases. Consanguinity is also a predictor ( $p$ value $=0.008$ ) of murmur on regression analysis.

Significant maternal history is associated with murmur in baby. In this study, 5 mothers have significant history; 3 mothers have history of epilepsy, none of these babies had murmur; 1 mother has history of pregnancy induced hypertension, baby was diagnosed as patent ductus arteriosus with secundum type of atrial septal defect; 1 mother has toxoplasma IgG positive, baby had murmur but echocardiography was normal.

$24(63 \%)$ out of 38 murmur cases were detected in babies less than 9 days. There is no association between age and presence of murmur. The mean age of presentation of murmur is 10 days; 6 babies were having patent ductus arteriosus (both more than $2 \mathrm{~mm}$ and less than $2 \mathrm{~mm}$ ) in less than 9 days' age group.

Three (7.8\%) babies were having dysmorphic features. One of the baby had bilateral ear tags, other had microophthalmia and one had low set ears, depressed nasal bridge, low hairline. Baby with micro-ophthalmia was diagnosed as endocardial cushion defect on echocardiography and further workup confirmed as congenital rubella syndrome. Echocardiography of other two babies with dysmorphic features was normal, though they had murmur.

$4(16 \%)$ cases required urgent cardiac intervention among babies with structurally significant heart lesion. They include single ventricle (1), tricuspid stenosis (1) and endocardial cushion defect (2).

\section{DISCUSSION}

Detection of a murmur on routine examination gives a clue to the presence of heart disease and offers the possibility of early presymptomatic diagnosis. Auscultation is, therefore, part of routine neonatal examination and is recommended in Health for All Children.(15)

In this study, 38 (3.9\%) newborns out of 977 newborns who underwent auscultation were found to have murmur. Characteristic of newborns having murmur and those not having murmur were studied. Out of 38 babies having murmur 17 (45\%) were female and 21 (55\%) were male. Distribution of male and female babies is similar in murmur 
cases. There is no association between sex and presence of murmur ( $p$ value- 1 , not significant).

The mean age of detecting murmur in this study is 10 days, it was 12 days in a study conducted by Mackie et al.(16) Out of 38 cases of murmur, 24 (63\%) newborns were of age less than 9 days. This may be because routine examination of early neonatal age was done more frequently. The mean birth weight of babies having murmur was $2.579 \mathrm{~kg}$, low birth weight $(<2.5 \mathrm{~kg})(\mathrm{p}=.006)$ was predictor of murmur in this study.

Prevalence of murmur in routine neonatal examination in our study was $3.9 \%$. After echocardiography evaluation, out of $38(3.9 \%)$ babies had murmur, $25(66 \%)$ babies were having structurally significant heart lesion, 8 (21\%) babies had structurally insignificant heart lesion, 5 (13\%) babies had normal report. The prevalence of structurally significant heart disease in our study was $66 \%$, which means if murmur was heard there was $66 \%$ cases who will have underlying cardiac malformation. In our study, prevalence of innocent murmurs was $1.3 \%$

Sean B Ainsworth(6) et al conducted a study, in which auscultation was done within 48 hours of delivery by house officers with exclusion criteria similar to our study, those with murmur underwent echocardiography within 24 hours. The prevalence of murmurs in routine neonatal examination was $0.6 \%$. The prevalence of structurally significant heart disease in this study was $54 \%$. In this study, $45 \%$ subjects had normal and structurally insignificant heart lesion as a group similar to our study.

A retrospective study carried out by Jill Gregory(17) et al of a cohort of 6 weeks old babies found 1\% of babies having murmur on routine examination at this age and $50 \%$ of murmur cases having structurally significant heart lesions. Another retrospective study carried out by Christopher Wren(18) et al included outcome of congenital heart diseases on the basis of examination at neonatal and 6 weeks examination, $67 \%$ patients were asymptomatic at birth. Routine examination was suggestive of abnormal finding only in $45 \%$ asymptomatic patients at birth. At 6 weeks followup examination $65 \%$ were having abnormal examination, but only $50 \%$ of discharged patients came for followup at 6 weeks; $30 \%$ of asymptomatic babies died of undiagnosed congenital heart disease before 6 weeks of age.

In a study conducted in India by $\mathrm{M}$ Bansal(19) et al, murmurs were detected in $2.3 \%$ of newborns on routine examination of whom $45 \%$ had cardiac malformation, $29 \%$ had an insignificant structural heart lesion and 25\% had completely normal echocardiogram. Similar study was done by Amer A Lardhi(20) in Saudi Arabia, in which routine examination was done within $24 \mathrm{hrs}$. of delivery with similar exclusion criteria of our study. Murmurs were detected in $1.37 \%$ babies. Echocardiography and Doppler study confirmed a cardiac malformation in $42.5 \%, 27 \%$ had an insignificant structural heart lesion, i.e. physiological variant that would account for a murmur such as (patent foramen ovale, small patent ductus arteriosus, mild peripheral pulmonary stenosis) and $22 \%$ had structurally normal hearts.

The reported prevalence of heart murmurs in neonates varies from $6 \%$ to $77.4 \%$.(20) In our study prevalence of murmurs was $3.9 \%$, which was on higher side as compared to other related studies done in various parts of the world. The reason for this may be in our study routine neonatal examination included newborns upto 3 days in case of normal delivery and 7 days in case of caesarean section deliveries. All the newborns upto 6 weeks who came for regular followup were also included in our study. In contrast studies mentioned earlier included newborns upto 2 - 3 days after birth. The frequency of examination in our study was more as compared to other studies. Prevalence of structurally significant heart lesions among cases of murmur in our study is similar to related studies described above. It is an important outcome to know that some subjects having murmur had completely normal echocardiogram when evaluated. Prevalence of murmur in our study is comparable to Indian study conducted by M Bansal et al.(19)

In our study among 38 cases of murmur 18 (48\%) cases had pansystolic murmur, 17 (44\%) cases had ejection systolic murmur and $3(8 \%)$ cases had continuous murmur. In a study conducted by Duangmanee Laohaprasitiporn(21) et al $90 \%$ were ejection systolic murmur, $8 \%$ were pansystolic murmur and $2 \%$ were continuous murmur.

Distribution of murmur as per location in our study was as follows: $12(32 \%)$ cases each; murmur was best heard at pulmonary area and upper left sternal border, in 10 (26\%) cases murmur was best heard at left lower sternal border and in only $4(10 \%)$ cases murmur was best heard at mitral area. Statistical analysis was suggestive of no correlation of location of murmur and structurally significant heart lesion. It means differentiation of pathological from innocent murmur is not possible on location of murmur in neonatal age group.

Grade 3 murmur (56\%) was most commonly heard, murmur during routine neonatal clinical examination. Structurally significant heart lesions were independent of grade of murmur on statistical analysis. It emphasises that all murmurs should be carefully evaluated in neonates and all low-grade murmurs are not innocent murmurs. In a study conducted by Duangmanee Laohaprasitiporn(21) et al, 65\% murmurs were less than grade 3 .

Acyanotic congenital heart diseases form $92 \%$ of structurally significant heart lesion detected on routine auscultation in neonates. In our study, classification pointed out that combined lesions form major part in this group followed by isolated lesions.

In isolated lesions, 3 cases were of VSD and 4 cases were of ASD. Out of four only one ASD was $10 \mathrm{~mm}$, remaining 3 were less than $8 \mathrm{~mm}$. In more than $80 \%$ cases, ASD of $3-8$ mm close spontaneously. ASD as part of combined lesions were of less haemodynamic significance, because pressure gradient and shunt across atria was insignificant.

VSD as an isolated lesion was present in only $3(12 \%)$ cases, but as a part of combined lesions with haemodynamic significance contributed to $8(32 \%)$ cases. VSD was contributing to $44 \%$ of significant lesions. Isolated PDA was present in only $2(8 \%)$ cases and was part of combined lesions in 5 (20\%) cases; 2 (8\%) patients had cyanotic type of congenital heart disease- single ventricle 1 (4\%) and tricuspid stenosis 1 (4\%).

In study conducted by D Laohaprasitiporn(21) et al in Thailand, three most common cardiac lesions were ventricular septal defect (18.4\%), atrial septal defect (16.3\%) and patent ductus arteriosus (16.3\%). In study conducted by Wren $\mathrm{AJ}(18)$ et al, the most common lesions found were those creating left-to-right shunts (66\%). Ventricular septal defect 
was the most common single lesion (37\%) followed by patent ductus arteriosus (23\%).

Prevalence of congenital heart disease in recent studies like Baltimore Washington study(22) is less than studies done previously by Mitchell et al, because recent studies have confirmed diagnosis on basis of echocardiography and catheterisation study. Pulmonary hypertension is part of diagnosis in $8(20 \%)$ cases in our study, which may be over estimation because Doppler method gives higher pulmonary artery pressure values.(12)

In $21 \%$ cases, $x$-ray was abnormal and suggestive of heart disease in babies who underwent evaluation of murmur. In our study, x-ray abnormality is not associated with structurally significant heart lesions. Electrocardiogram was abnormal only in $16 \%$ cases in babies who underwent evaluation of murmur. Abnormal ECG is also not associated with structurally significant heart lesions. In many related studies, they had similar results. In a study conducted by Duangmanee Laohaprasitiporn et al,(21) chest roentgenography and electrocardiography are used as initial tools in diagnosis of CHD. In the present study, the majority of CHD patients had normal results with normal chest roentgenogram in $79.6 \%$ and normal electrocardiogram in $75.5 \%$. Andrew Mackie(16) et al conducted study to determine the clinical features that predict the presence of congenital heart disease (CHD) in this population. The addition of an electrocardiogram did not improve these test characteristics.

In this study, family history of congenital heart disease was present in $2(5.2 \%)$ of newborns with murmur with significant association with presence of murmur. Risk of having congenital heart disease increases 3 - 8 times with one parent or sibling is affected. When two first degree relatives are affected, risk may reach $30 \%$.(1) A Settin(23) et al conducted study in patients of congenital heart disease with significant correlation of positive family history.

Consanguinity and presence of murmur was significant association; it was also a strong predictor of murmur in newborn in this study. In this study in $4(10.5 \%)$ neonates with murmur, there was history of second degree consanguineous marriage of parents. Cause of most congenital heart defects is unknown. Most cases are multifactorial and result from combination of genetic and environmental factors. Specific chromosomal and gene defects are associated with congenital heart lesions in various syndromes. ${ }^{(2)}$

Study done by Yunis $\mathrm{K}$ et al(24) concluded that consanguinity remained significantly associated with an increased risk of CHD: Infants born to first cousin marriages had a 1.8 times higher risk of having a CHD diagnosed at birth compared to those born to unrelated parents. Consanguinity was a significant risk factor for ventricular septal defect (VSD), atrial septal defect (ASD), hypoplastic left heart (HLH), and single ventricle (SV).

Significant maternal history is present in $2 \%$ to $4 \%$ of cases of congenital heart disease, e.g. maternal diabetes, congenital rubella syndrome, drugs (lithium, alcohol, warfarin, thalidomide, anticonvulsants).(1) In this study 2 (5\%) newborns with murmur had significant maternal history, one case had pregnancy induced hypertension and maternal toxoplasma immunoglobulin G reactivity in other. The association between maternal history and murmur was statistically significant.
Cyanosis is a sign which needs urgent evaluation and were admitted in neonatal intensive care unit, such cases were excluded from this study. In our study 2 cases of cyanosis were detected after detection of murmur, which suggests that murmur can detect cyanotic congenital heart diseases in which cyanosis was subclinical and picked up on pulse oximeter. In our study, the cyanotic congenital heart diseases detected were single ventricle (4\%) and tricuspid stenosis $(4 \%)$ representing $8 \%$ of structurally significant heart disease among asymptomatic babies with murmur.

Majority of patients with congenital anomaly were seriously ill and were excluded from this study. In this study, $1(2.6 \%)$ patient had unilateral cleft lip. Association between murmur and cleft lip was not statistically significant, but congenital anomaly like cleft lip was predictor of murmur. Dysmorphic features were present in 3 patients as 1. Ear tags, 2. Micro-ophthalmia and 3. Low set ears, Low hairline, Depressed nasal bridge. Patient with micro-ophthalmia turned out as congenital rubella syndrome. Dysmorphic features were significantly related to murmur in a baby. A. Settin ${ }^{(23)}$ et al conducted a study related to this and concluded that presence of significant higher frequency of external dysmorphic features among studied cases with congenital heart disease than general population is probably indicative of the importance of these landmarks for early diagnosis of these cases.

In our study, $4(16 \%)$ cases required urgent cardiac intervention. They include single ventricle, tricuspid stenosis, endocardial cushion defect. In study conducted by A Lardhi(20) et al, $5.4 \%$ of neonates required early cardiac intervention before they became symptomatic. In a study conducted by Sean B Ainsworth(6) et al, 9\% of babies with murmurs required early cardiac surgery and were detected before they became symptomatic.

\section{CONCLUSION}

Unrecognised neonatal heart disease carries a serious risk of avoidable mortality, morbidity and handicap. Early detection of murmur and intervention is important to prevent worsening and complications in cases of congenital heart disease. However, the absence of murmur does not exclude the presence of potentially serious heart disease. Diagnosis of innocent murmurs is also important to exclude cardiac disease and reassurance of parents.

Finally, the take home message is in developing country like India it is not possible to screen every newborn for presence of heart disease at birth; hence, routine clinical assessment should be thoroughly and sincerely performed at birth and before discharge to pick up any murmur which could be suggestive of underlying heart disease. This will definitely help to curtail down the mortality and more importantly the morbidity associated with heart disease in children.

\section{REFERENCES}

[1] Perloff JK. Clinical recognition of congenital heart disease. $5^{\text {th }}$ edn. WB Saunders 2003.

[2] Behrman RE, Kliegman RM, Jenson HB. Nelson Textbook of Pediatrics. 18 $8^{\text {th }}$ edn. W B Saunders 2007.

[3] Abu-Harb M, Hey E, Wren C. Death in infancy from unrecognised congenital heart disease. Arch Dis Child 1994;71(1):3-7. 
[4] Gandy GM. Examination of the neonate including gestational age assessment. In: Roberton NR. edr. Textbook of Neonatology. 2nd edn. Edinburgh: Churchill Livingstone 1992.

[5] Rudolph AM. The changes in the circulation after birth. Their importance in congenital heart disease. Circulation 1970;41(2):343-59.

[6] Ainsworth SB, Wyllie JP, Wren C. Prevalence and clinical significance of cardiac murmur in neonates. Arch Dis Child Fetal Neonatal Ed 1999;80(1):F43-5.

[7] Lyon RA, Rauh LW, Stirling JW. Heart murmurs in newborn infants. J Pediatr 1940;16(3):310-7.

[8] Saxena A. Congenital heart disease in India: a status report. Indian Journal of Pediatrics 2005;72(7):595-8.

[9] Rubin JD, Ferencz C, Brenner JL, et al. Early detection of congenital cardiovascular malformations in infancy. Am J Dis Child 1987;141(11):1218-20.

[10] Swash M. Hutchison's clinical methods. 21 st edn. Saunders 2002.

[11] Mitchell SC, Korones SB, Berendes HW. Congenital heart disease in 56, 109 births. Incidence and natural history. Circulation 1971;43(3):323-32.

[12] Park MK. Pediatric cardiology for practitioners. $5^{\text {th }}$ edn. New Delhi: Mosby Elsevier 2007.

[13] Arlettaz R, Archer N, Wilkinson AR. Natural history of innocent heart murmurs in newborn babies: controlled echocardiographic study. Arch Dis Child Fetal Neonatal Ed 1998;78(3):166-70.

[14] Mullins CE, Pagotto L. Patent ductus arteriosus. In: Garson A, Bricker JT, Fisher DJ, et al. eds. The science and practice of pediatric cardiology. $2^{\text {nd }}$ edn. Baltimore: Williams \& Wilkins 1998:1181-97.

[15] Hall DMB. Health for All Children. Report of the Third Joint Working Party on Child Health Surveillance. Oxford University Press, Oxford 1996.
[16] Mackie AS, Jutras LC, Dancea AB, et al. Can cardiologists distinguish innocent from pathologic murmurs in neonates? The Journal of Pediatrics 2009;154(1):50-4.e1.

[17] Gregory J, Emslie A, Wyllie J, et al. Examination for cardiac malformations at six weeks of age. Arch Dis Child Fetal Neonatal Ed 1999;80(1):F46-F8.

[18] Wren C, Richmond S, Donaldson L. Presentation of congenital heart disease in infancy: implications for routine examination. Arch Dis Child Fetal Neonatal Ed 1999; 80(1):F49-F3.

[19] Bansal M, Jain H. Cardiac murmurs in neonate. Indian Journal of Pediatrics 2005;42:397-8.

[20] Lardhi AA. Prevalence and clinical significance of heart murmurs detected in routine neonatal examination. Journal of the Saudi Heart Association 2010;22(1): 25-7.

[21] Laohaprasitiporn D, Jiarakamolchuen T, Chanthong P, et al. Heart Murmur in the first week of life: Siriraj Hospital. J Med Assoc Thai 2005;88(8):S163-8.

[22] Ferencz C, Rubin JD, McCarter RJ, et al. Congenital heart disease: prevalence at livebirth. The BaltimoreWashington Infant Study. American Journal of Epidemiology 1985;121(1):31-6.

[23] Settin A, AlMarsafawy H, AlHussieny A, et al. Dysmorphic features, consanguinity and cytogenetic pattern of congenital heart diseases: a pilot study from Mansoura Locality, Egypt. Int J Health Sci (Qassim) 2008;2(2):101-11.

[24] Yunis K, Mumtaz G, Bitar F, et al. Consanguineous marriage and congenital heart defects: a case-control study in the neonatal period. Am J Med Genet A 2006;140(14):1524-30. 